\title{
A Multicenter, Double-Blind, Phase III Clinical Trial to Evaluate the Efficacy and Safety of a Cell and Gene Therapy in Knee Osteoarthritis Patients
}

\author{
Myung-Ku Kim, ${ }^{1, \dagger}$ Chul-Won $\mathrm{Ha}^{2, \dagger}$ Yong In, ${ }^{3}$ Sung-Do Cho, Eui-Sung Choi, \\ Jeong-Ku Ha, Ju-Hong Lee, Jae-Doo Yoo, Seong-II Bin, \\ Choong-Hyeok Choi, ${ }^{10}$ Hee-Soo Kyung, ${ }^{11}$ and Myung-Chul Lee ${ }^{12, *}$
}

\footnotetext{
${ }^{1}$ Inha University Hospital, Incheon, South Korea; ${ }^{2}$ Samsung Medical Center, Sung Kyun Kwan University School of Medicine, Seoul, South Korea; ${ }^{3}$ The Catholic University of Korea College of Medicine, Seoul, South Korea; ${ }^{4}$ Ulsan University Hospital and ${ }^{9}$ Asan Medical Center, University of Ulsan College of Medicine, Ulsan, South Korea; ${ }^{5}$ Chungbuk National University Hospital, Cheongju, South Korea; ${ }^{6}$ Seoul Paik Hospital, Inje University, Seoul, South Korea; ${ }^{7}$ Jeonbuk National University Medical School, Jeonju, South Korea; ${ }^{8}$ Ewha Womans University Mokdong Hospital, Seoul, South Korea; ${ }^{10}$ College of Medicine, Hanyang University, Seoul, South Korea; ${ }^{11}$ Kyungpook National University, Daegu, South Korea; ${ }^{12}$ Seoul National University Hospital, Seoul, South Korea.

†These authors contributed equally to this work.
}

The aim of this study was to test the clinical efficacy of TissueGene-C (TG-C), a cell and gene therapeutic for osteoarthritis consisting of non-transformed and transduced chondrocytes (3:1) retrovirally transduced to overexpress transforming growth factor- $\beta 1$. A total of 163 Kellgren-Lawrence grade 3 patients with knee osteoarthritis were randomly assigned to receive intra-articular TG-C or placebo. Primary efficacy measures included criteria for subjective assessment by International Knee Documentation Committee (IKDC) and pain severity by Visual Analog Scale (VAS) for 52 weeks. Secondary efficacy measures included IKDC and VAS at 26 and 39 weeks; pain, stiffness, and physical function by the Western Ontario and McMaster Universities Arthritis Index (WOMAC); and pain, symptoms, daily activities, function in sports and recreation, and quality of life by the Knee Injury and Osteoarthritis Outcome Score (KOOS), X-ray, magnetic resonance imaging, and soluble urine and blood biomarkers. TG-C was associated with statistically significant improvement over placebo in the total IKDC score and individual categories, and in the VAS score at 26, 39, and 52 weeks. WOMAC and KOOS scores also improved with TG-C over placebo. Patients treated with TG-C showed trends directed toward thicker cartilage and slower growing rates of subchondral bone surface area in the medial tibia, lateral tibia, lateral patella, and lateral patella femoral regions, although these were not statistically significant $(p>0.05)$. Serum C-terminal telopeptide of type I collagen (CTX-I) and urine CTX-II levels were lower over 1 year in TG-C than placebo-treated patients, with CTX-I level reaching statistical significance. These tendencies supported TG-C as holding great potential as a disease-modifying osteoarthritis drug. The most frequent adverse events in the TG-C group were peripheral edema (9\%), arthralgia (8\%), joint swelling $(6 \%)$, and injection site pain (5\%). TG-C was associated with statistically significant improvements in function and pain in patients with knee osteoarthritis. The unexpected adverse events were not observed.

Keywords: TissueGene-C, osteoarthritis, gene therapy, Phase III

\section{INTRODUCTION}

OSTEOARTHRITIS IS A PROGRESSIVE, degenerative disease that affects more than 250 million people worldwide, and in the absence of efficacious new disease-modifying osteoarthritis drugs (DMOADs), the prevalence of osteoarthritis is expected to increase rapidly as the global population ages and obesity rates increase. ${ }^{1,2}$ Moreover, as a leading cause of physical disability around the world, osteoarthritis places a heavy burden on global healthcare expenditure. ${ }^{3}$ Treatment of osteoarthritis, which most often afflicts weight-bearing knee and hip joints, predominantly entails symptom relief, with nonsteroidal anti-inflammatory drugs being

${ }^{*}$ Correspondence: Dr. Myung-Chul Lee, Department of Orthopedic Surgery, Seoul National University Hospital, Seoul, South Korea. E-mail: leemc@snu.ac.kr 
the most commonly prescribed agents. However, these treatments are often ineffective at relieving pain and can produce unwanted side effects and fail to halt disease progression, especially for the patient demographic groups in which osteoarthritis is prevalent. ${ }^{4}$ Therefore, a growing unmet need exists for $\mathrm{DMOADs}$ to slow or reverse the course of disease progression and provide long-lasting benefits to osteoarthritis patients.

Chondrocytes maintain the integrity of articular cartilage by controlling the production and degradation of extracellular matrix components, including proteoglycans and collagens. ${ }^{5}$ In osteoarthritis, this equilibrium is disrupted such that a net degradation of articular cartilage occurs with increased production of inflammatory cytokines and matrix-degrading enzymes. Many ongoing drug-development strategies for osteoarthritis aim to slow or reverse cartilage degeneration by regulating chondrocyte function. Transforming growth factor- $\beta$ (TGF- $\beta$ ), which is an anti-inflammatory cytokine, plays a key role in maintaining metabolic homeostasis and the structural integrity of articular cartilage by controlling chondrocyte proliferation and differentiation, as well as extracellular matrix deposition. ${ }^{6-8}$ TGF- $\beta$ has therefore been considered a primary candidate for restoring damaged cartilage in the articular joints of osteoarthritis patients. Fibroblast growth factor 18 (FGF-18), acting via FGF receptor-3, promotes chondrocyte proliferation, extracellular matrix formation, and cartilage regeneration. ${ }^{9} \mathrm{~A}$ recombinant form of human FGF-18 has shown promise as a DMOAD in early clinical trials. ${ }^{10,11}$ Cell-based strategies are being developed to overcome problems associated with growth factor pharmacokinetics. Mesenchymal stem cells are multipotent and can develop into cartilage and bone, possess antiinflammatory and immunosuppressive activities, and have shown promise for osteoarthritis treatment in early clinical studies. ${ }^{12,13}$ Autologous chondrocyte transplantation has shown some promise in initial clinical studies for full-thickness chondral defects of the knee, despite concerns regarding the potential for chondrocyte dedifferentiation. ${ }^{12,14}$

TissueGene-C (TG-C) was developed as a cellbased growth factor expression strategy and involves a 3:1 ratio of human allogeneic chondrocytes and genetically modified chondrocytes engineered to express TGF- $\beta 1$. TGF- $\beta 1$ expressing cells were irradiated with a low dose of gamma-ray so that they could not proliferate within the knee joint after injection. TG-C was administered by a single intra-articular injection. ${ }^{15,16}$ In Phase II trials of TG-C in patients with knee osteoarthritis, the intra-articular injection was well tolerated and was shown to improve functionality, pain, and quality of life when compared to patients either at the start of treatment or given a placebo. ${ }^{17,18}$ Here, the results are reported of a placebo-controlled, doubleblind, randomized, parallel group, 12 site, Phase 3 trial with TG-C in patients with osteoarthritis of the knee.

\section{MATERIALS AND METHODS}

\section{Study population}

Eligible patients aged $\geq 19$ years were enrolled who had osteoarthritis of the knee diagnosed by an International Knee Documentation Committee (IKDC), ${ }^{19}$ with a subjective score of $\leq 60$ (100-point scale), a $100 \mathrm{~mm}$ Visual Analog Scale (VAS) ${ }^{20}$ pain score of $\geq 40 \mathrm{~mm}$, radiographic confirmation of grade 3 osteoarthritis on the Kellgren-Lawrence scale, ${ }^{21}$ and International Cartilage Repair Society (ICRS) ${ }^{22}$ grade 3 or 4 cartilage damage in major lesions, as confirmed by magnetic resonance imaging (MRI; per the inclusion/exclusion criteria shown in Supplementary Table S1; Supplementary Data are available online at www.liebertpub .com/humc). Four patients were withdrawn from this study prior to receiving any treatment: three due to withdrawal of consent and one due to not meeting the inclusion/exclusion criteria; Fig. 1). The study adheres to the CONSORT statement, and a flow diagram is outlined in Fig. 1.

To avoid bias, an independent person prepared a randomization table using a random permuted block design. Random allocation numbers were assigned to investigational agents before they were shipped to the study site. A randomization number was assigned to each patient, and a drug with the same serial number was administered. Investigator blinding was achieved by an independent person who administered TG-C or placebo as per the assigned randomization number. Patient blinding was achieved by filling investigational agents in the same color syringes.

Study-eligible patients were treated with a single $3 \mathrm{~mL}$ injection of TG-C, which was pooled into one syringe from two components of non-transduced human allogeneic chondrocytes (hChonJ; $1.35 \times 10^{7}$ cells $/ 2 \mathrm{~mL}$ ) and transduced human allogeneic chondrocytes (hChonJb\#7; $4.5 \times 10^{6}$ cells $/ 1 \mathrm{~mL}$ ) or a placebo (saline). The injection method used was identical to standard knee injection treatments, such as with hyaluronic acid and/or steroids.

\section{Efficacy evaluations}

Primary endpoints. The effect of TG-C on the subjective IKDC assessment score was assessed, 


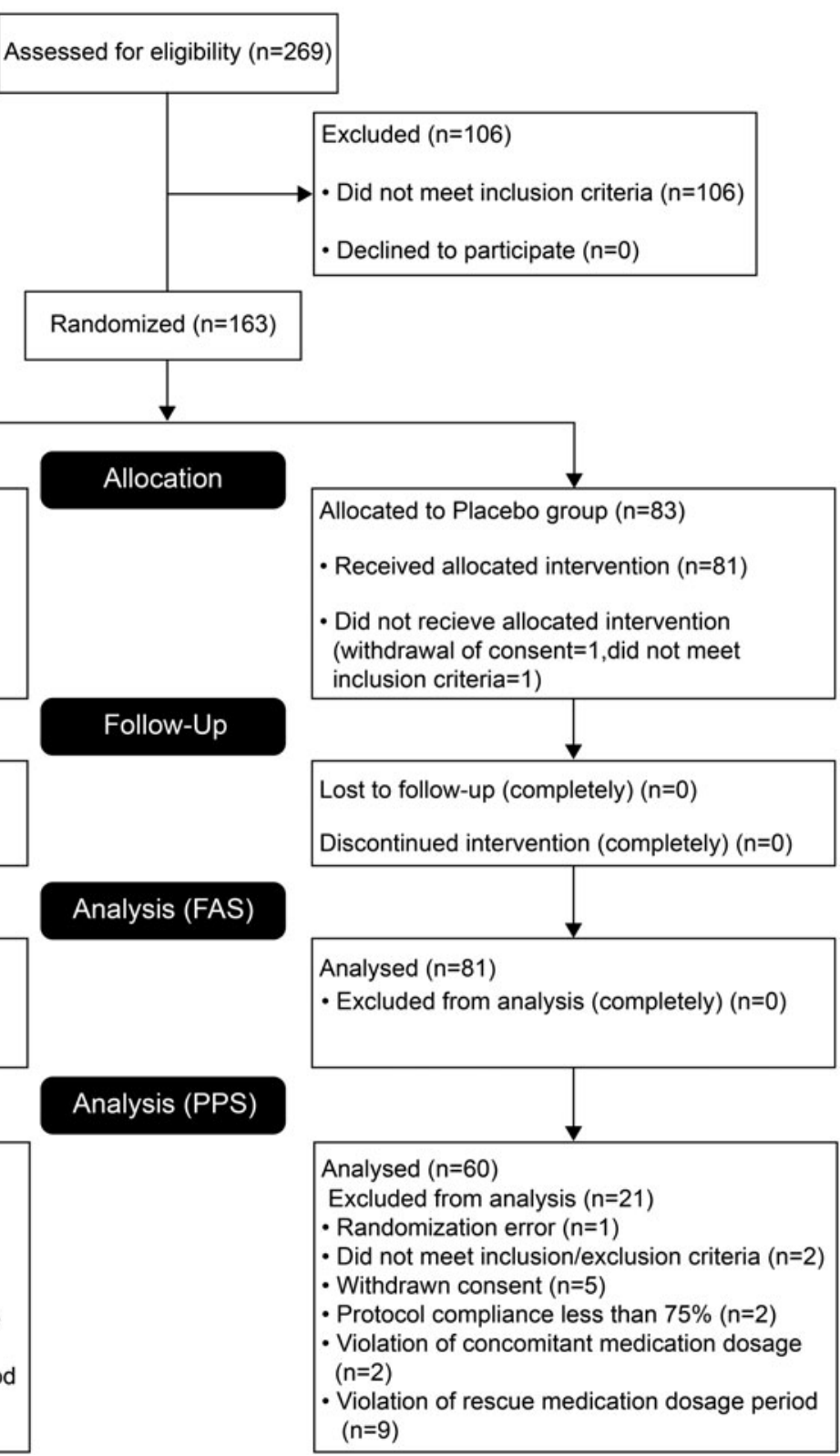

Figure 1. Flow diagram of patient enrollment and analysis. The full analysis set (FAS) was used for analyzing both the efficacy and safety endpoints.

with the outcomes transformed to a 100-point score (higher scores corresponded to better function/ fewer symptoms), and changes in pain severity represented by a $100 \mathrm{~mm}$ VAS score (0-100 points; lower scores corresponded to less pain) at 52 weeks (12 months) post injection compared to baseline.

Secondary endpoints. The effect of TG-C on IKDC and $100 \mathrm{~mm}$ VAS scores at 26 (6 months) and 39 weeks (9 months) post injection were assessed compared to baseline. The effect of TG-C on the Western Ontario and McMaster Universities Arthritis Index (WOMAC) score $^{23}$ (measuring pain, stiffness, and physical function), the Knee Injury and Osteoarthritis Outcome Score (KOOS) score ${ }^{24}$ (100-point scale measuring pain, symptoms, and activities in daily living [ADL], knee function in sport and recreation, and knee-related quality of life) was also assessed at 26, 39, and 52 weeks post injection. Changes in joint space narrowing were measured by X-ray imaging in the posteroanterior view using a SynaFlexer ${ }^{\mathrm{TM}}$ positioning frame ${ }^{25}$ (Synarc, Newark, CA). Whole-organ MRI scoring (WORMS) was used to incorporate changes in edema lesion scoring, cartilage thickness defect depths, cartilage defect surface areas, osteophytes, 
joint fluids, meniscus structures, meniscus signals, periarticular inflammation, and synovial thickening and inflammation. Finally, the ICRS grade at 26 and 52 weeks was used to assess cartilage improvement based on the size of the major lesion.

Additional quantitative evaluations of MRI changes were conducted in cartilage thickness and subchondral bone areas. Quantitative changes were evaluated by measuring the bone area of all anatomical regions in the femur, tibia, and patel$\mathrm{la}^{26}$ and by automated segmentation of the hyaline cartilage in Double Echo Steady State (DESS) images (or similar images). ${ }^{27}$ Consistent measurements of thickness were then taken using the active appearance model (AAM; a type of shape model designed for examining fat-saturated, highresolution images such as the Siemens DESS-we sequence). AAM imaging produces detailed and consistent maps of cartilage thicknesses for the entire femur, tibia, and patella cartilage surfaces, subdivided into anatomical masks, which was previously accomplished by manual segmentation. ${ }^{28,29}$

Changes in the central medial femur (cMF) region and the central medial tibia (cMT) region were measured. These regions have been identified by various investigators (including some at Imorphics) as the most responsive regions for detecting $\mathrm{OA}$ progression. ${ }^{30}$ The average cartilage thickness was measured from the subchondral bone $^{31}$ within a defined anatomical bone region, and is reported here as an average over the region in millimeters.

The effect of TG-C was assessed on serum C-terminal telopeptide of type I collagen (CTX-I, a subchondral bone resorption marker), serum cartilage oligomeric matrix protein (COMP), procollagen type II N-terminal propeptide (PIIANP), markers of cartilage joint metabolism, matrix metalloproteinase 3 (MMP-3), C1M and C3M (markers of tissue inflammation at the synovial membrane), C2M, CS846, urine biomarkers carboxy-terminal of cross-linked fragment type II collagen (CTX-II), and the CTX-II/creatinine ratio at 26,39 , and 52 weeks post injection.

\section{Safety evaluations}

The onset, nature, duration, severity, relationship to the study treatment, and outcome of all adverse events were determined and documented during each visit (4, 12, 26, 39, 52 weeks). Safety evaluations included physical examination (vital signs, pulse, temperature, systolic blood pressure, and diastolic blood pressure), laboratory test results (normal/abnormal changes in hematology, serum chemistry, and urine testing), allergic reactions (common terminology criteria for adverse events [CTCAE v4.02] grade 4 or higher), injection site reaction and infection at the injection site, fibrosis-cosmesis, fibrosis in deep connective tissue, muscular/skeletal hypoplasia (all CTCAE grade 3 or higher), and TGF- $\beta 1$ enzyme-linked immunosorbent assay (ELISA) test results (above the normal range of $\sim 65 \mathrm{ng} / \mathrm{mL}$ ) during screening and at 52 weeks.

\section{Statistical analysis}

To maintain the overall statistical power for the two common endpoints (IKDC and $100 \mathrm{~mm}$ VAS) at $\geq 80 \%$ or above, the test power for each endpoint was set at $90 \%$. The number of patients calculated was 78 per group for the IKDC endpoint and 31 per group for the $100 \mathrm{~mm}$ VAS endpoint. Therefore, the plan was to enroll 78 patients per group, using the larger number needed to achieve significance for the IKDC endpoint.

Two-sample $t$-testing or Wilcoxon's rank-sum testing was performed per the normality test result to compare the level of improvement in primary endpoints of the IKDC total score and $100 \mathrm{~mm}$ VAS score in the TG-C and placebo groups. Analysis of covariance testing was conducted using the TG-C group and the pre-dose baseline IKDC or $100 \mathrm{~mm}$ VAS score as covariates, and regression analysis was performed for the endpoints showing statistically significant differences in the intergroup tests for demographic and medical history data. In addition, an intergroup test was performed using a method of analyzing data repeatedly measured through normality evaluation (repeated-measure analysis of variance [ANOVA] testing), and the significance level was set at $5 \%$.

For the secondary efficacy endpoints, the continuous data were compared by the two-sample $t$-test or Wilcoxon's rank-sum test per the normality test result, and repeatedly measured data were tested using a repeated-measures ANOVA test. Categorical data were tested by the chi-square test or Fisher's exact test, depending on the expected frequency of each cell. The chi-square test or Fisher's exact test was also used for intergroup comparisons of normal and abnormal results following physical examination and for laboratory tests. The incidence, confidence interval (CI), and incidence frequency of adverse events, treatment-related events, and serious adverse events were calculated, and intergroup comparisons of incidences were performed using the chi-square test or Fisher's exact test, depending on the expected frequency of cells. Allergic reaction (CTCAE grade 4 or higher), injection site reaction 
(CTCAE grade 3 or higher), infection (CTCAE grade 3 or higher), fibrosis-cosmesis (CTCAE grade 3 or higher), fibrosis-deep connective tissue (CTCAE grade 3 or higher), and muscular/skeletal hypoplasia (CTCAE grade 3 or higher) were confirmed by presenting the incidence rate and number of cases occurring per system organ class/preferred term (SOC/ PT), based on the severity of adverse events. Patients deviating from the normal range (TGF- $\beta 1$ ELISA test score of $\sim 65 / \mathrm{mL}$ ) were confirmed by performing intra- and intergroup comparisons for each visit.

Imorphics performed additional image analysis for various bone area regions and cartilage thickness-measurement regions. Statistical significance between the TG-C and placebo groups was evaluated using Student's $t$-test. Graphs of the results were produced, with error bars representing the CIs. Bone area changes were normalized to knee widths to correct for differences in patient size.

\section{Study approval}

The study protocol, informed consent form, patient information sheet, and other study-related documents were pre-approved by Ministry of Food and Drug Safety and the Institutional Review Board of each study center. Written informed consent was received from participants prior to inclusion in the study. The procedures followed were in accordance with the ethical standards of the responsible committee on human experimentation (institutional and national) and with the Declaration of Helsinki.

\section{RESULTS}

\section{Patient disposition, demographics,} and baseline characteristics

Of the 269 patients screened, 163 were randomized for this study, of which 159 were included in the full analysis set (four patients were excluded due to failure to take the investigational product; Fig. 1). Patients were $18 \%$ male and $82 \%$ female, with a mean age of $61.5 \pm 7.9$ years and a mean body mass index (BMI) of $25.6 \pm 2.6 \mathrm{~kg} / \mathrm{m}^{2}$. The mean duration of osteoarthritis in the patients was 5.4 years, with the main lesion sites being the left knee $(52 \%)$ and the right knee (48\%). The TG-C and placebo groups were not different with respect to the target disease, main lesion sites, medical history, and present illnesses (Supplementary Table S2).

\section{Efficacy}

Primary efficacy endpoints. Compared to the placebo, TG-C treatment was associated with a significantly greater improvement in IKDC score $(+15$ vs. +5 points; $p<0.001)$ and $100 \mathrm{~mm}$ VAS score $(-25$ vs. -10 points; $p<0.001)$ at 52 weeks versus baseline (Fig. 2).

Secondary efficacy endpoints. Compared to the placebo, TG-C treatment showed significant improvements in IKDC and VAS scores at 26 weeks (IKDC: $p=0.01$; VAS: $p=0.02$ ) and 39 weeks (IKDC: $p=0.02$; VAS: $p=0.004$ ) versus baseline. Improvements in the total WOMAC score were greater in the TG-C group than in the placebo group at 26 and 39 weeks, and significantly greater at 52 weeks $(p=0.003)$. TG-C treatment showed greater improvement in each WOMAC category of pain, stiffness, and physical function than did the placebo at each time point. Statistically significant differences were seen in all categories at 52 weeks (pain: $p<0.001$; stiffness: $p=0.003$; physical function: $p=0.008)$ and in pain at 26 weeks $(p=0.02)$ and 39 weeks $(p=0.02)$. A statistically significant improvement in the total KOOS score was noted with TG-C treatment compared to placebo at 26 weeks $(p=0.003), 39$ weeks $(p=0.03)$, and 52 weeks ( $p<0.001$; Fig. 2$)$. TG-C treatment showed a higher level of improvement in each KOOS category of pain, symptoms, ADL function, function in sports and recreation, and knee-related quality of life than did the placebo at each time point. Statistically significant differences were observed in pain $(p=0.002)$, symptoms $(p=0.005)$, ADL functions $(p=0.02)$, and functions in sports and recreation ( $p=0.03$ ) at 26 weeks, although a statistically significant difference was only observed in pain $(p=0.003)$ at 39 weeks. Significant differences were observed again in all categories at 52 weeks (pain: $p<0.001$; symptoms: $p<0.001$; ADL function: $p=0.002$; function in sports and recreation: $p=0.004$; quality of life: $p<0.001$ ).

Changes in joint space narrowing were measured by X-ray image analysis at 26 and 52 weeks, and were not significantly different between groups. Changes in bone marrow edema lesions, cartilage defect surface areas, joint fluids, meniscus structures, meniscus signals, periarticular inflammation, synovial thickening and inflammation, and sizes of major lesions were measured by MRI analysis using WORMS scoring at 26 and 52 weeks. Results showed that they were not significantly different between groups. However, osteophytes in the trochlea and patella regions in the placebo group showed a significant increase from baseline compared to those in the TG-C-treated group (Supplementary Table S3). Additional quantitative analysis with MRI data showed that the medial and lateral 
A Changes in IKDC: $P=0.01, P=0.02$, and $P<0.001$ at 26,39 and 52 weeks, respectively

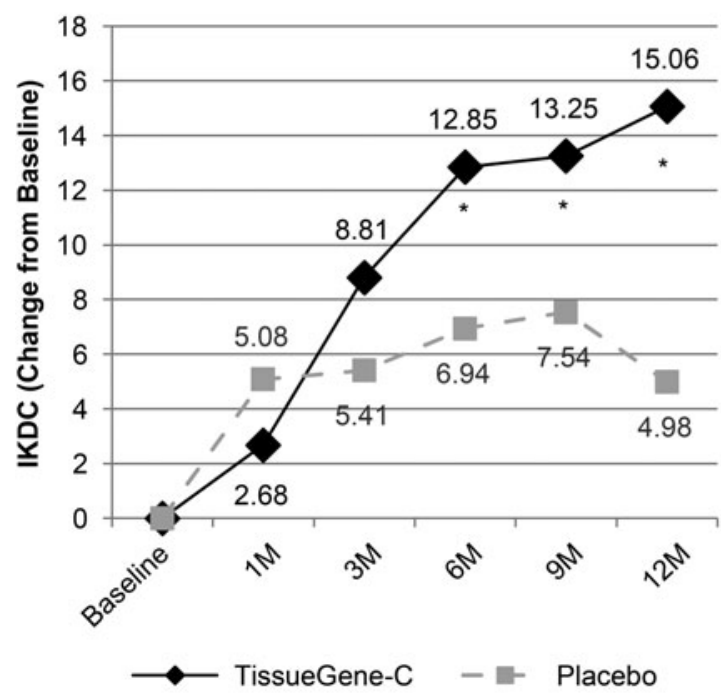

C Changes in WOMAC Score: $\mathrm{P}=0.003$ at 52 weeks

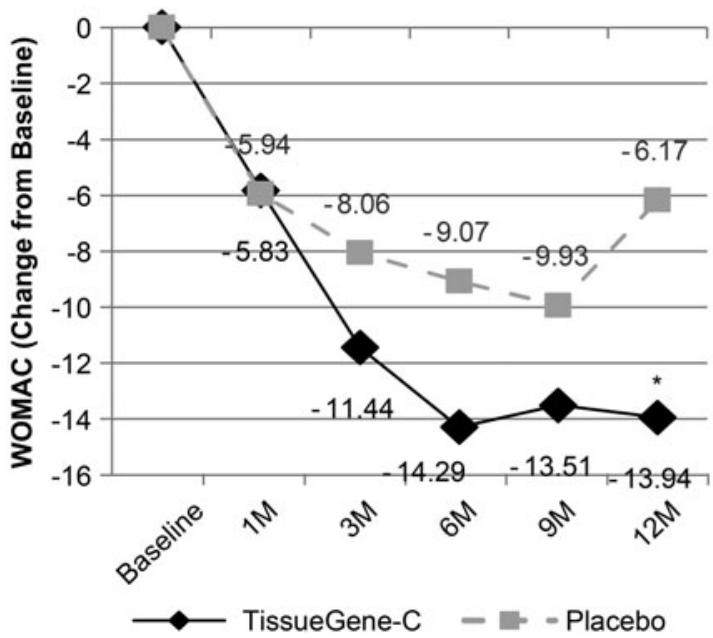

B Changes in VAS: $P=0.02, P=0.004$, and $P<0.001$ at 26,39 and 52 weeks, respectively

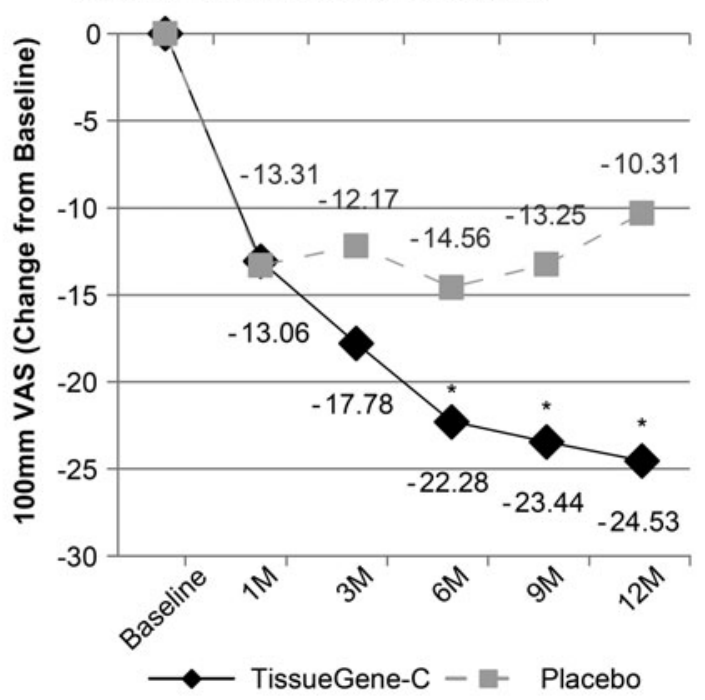

D Changes in KOOS: $P=0.003, P=0.03$, and $P<0.001$ at 26,39 and 52 weeks, respectively

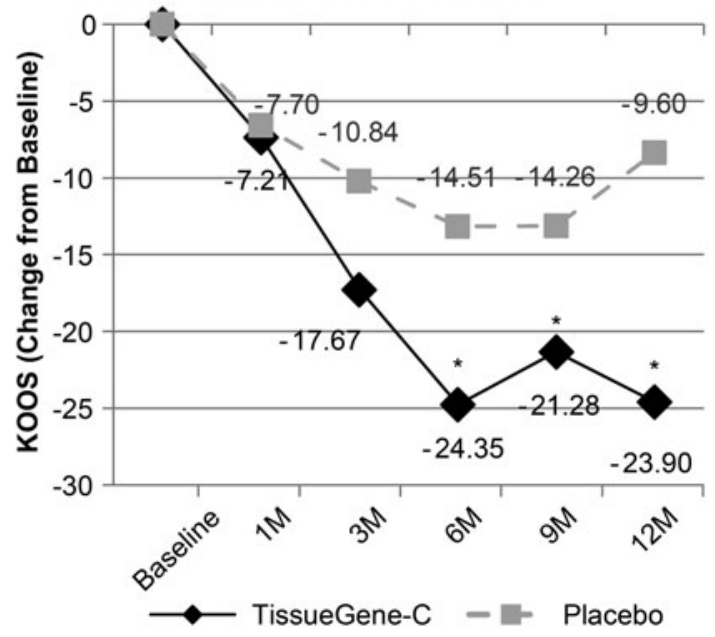

Figure 2. Improvements in efficacy endpoints with TissueGene-C.

tibial regions underwent a reduction in the rate of bone area change in TG-C group (Fig. 3). The lateral patella and lateral patella femoral regions showed clear trends toward reduced changes from 26 to 52 weeks, and in all regions, less change (but not statistically significant) occurred in patients treated with TG-C than the placebo (Fig. 4). At 52 weeks, both the mean tibia and femur cartilage thicknesses increased after TG-C treatment, although the differences were not statistically significant, while the placebo were not different from baseline (Fig. 4).

The levels of serum CTX-I were lower in the TG$\mathrm{C}$ group than in the placebo at all time points, with a significant decrease $(p=0.05)$ observed at 39 weeks. The levels of serum C1M $(p=0.03)$ and C3M $(p=0.02)$ were transiently significantly higher in the TG-C group than in the placebo group at 26 weeks, but not at later time points. The urine CTX$\mathrm{II} /$ creatinine ratio was lower in the TG-C group than in the placebo group at all time points, but the differences were not statistically significant. Other biomarkers, such as serum CS846, MMP-3 (placebo $<$ baseline), C2M, and PIIANP showed nonstatistical differences (both groups at 26 weeks $<$ baseline; Fig. 5). The frequency of rescue drug use was not significantly different between the groups, and no patients withdrew during the study for arthroplasty.

\section{Safety}

The rates of adverse events were $63 \%$ and $44 \%$ in the TG-C and placebo groups, respectively. The 
A
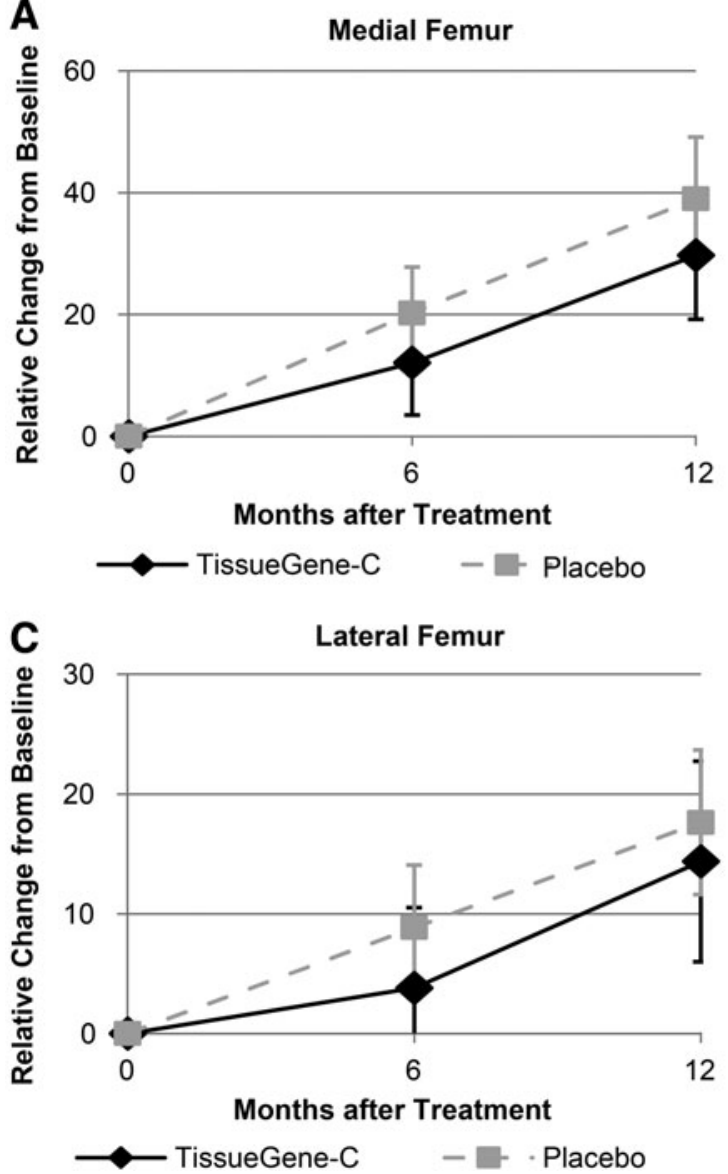

B
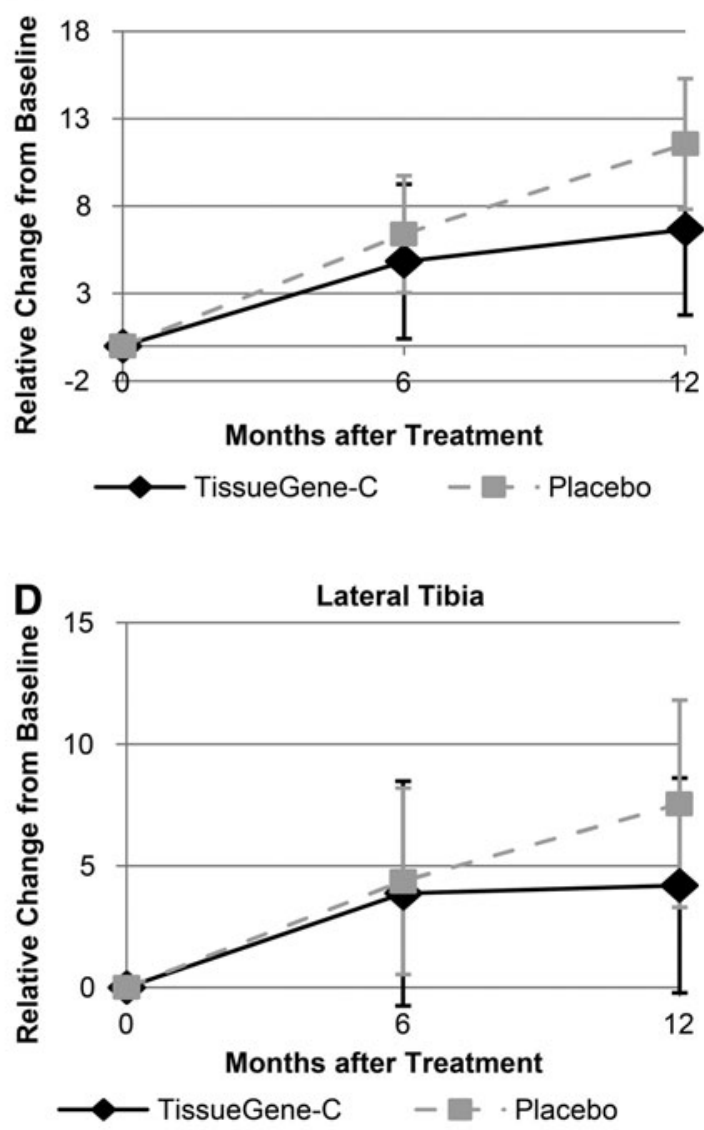

Figure 3. Primary bone area analysis. Medial femoral, medial tibial, lateral femoral, and lateral tibial regions. These figures show normalized changes from the baseline in the placebo and TissueGene- $C$ groups. Both the medial and lateral tibial regions showed a slowing rate of bone area changes in the TissueGene- $C$ group.

most common adverse events were arthralgia (occurring in $8 \%$ of patients in the TG-C group and $9 \%$ in the placebo group), peripheral edema (in $9 \%$ of patients in the TG-C and $1 \%$ in the placebo group), joint swelling (in $6 \%$ of patients in both groups), and injection site pain (in 5\% of patients in the TG$\mathrm{C}$ group; Table 1). Adverse events were resolved during the study period, except for three cases (joint swelling; one in the TG-C group and two in the placebo group), which were grade 1 adverse events. No categories other than injection site adverse events were reported as CTCAE grade 3 (severe) or higher. Six cases of adverse events at injection sites occurring in three patients in the TG-C group were assessed to be possibly related or related to TG-C and were resolved during the study period.

Serious adverse events occurred in 11 patients in the TG-C group: one patient each reported bladder cancer, carotid artery stenosis, multiple fractures, a mass of the right parotid, a suicide attempt, right knee medial meniscus tearing, nasal bone fracture, cerebral hemorrhage and left arm fracture, left knee swelling and pain (arthralgia), clavicle fracture, and itching on the trunk. Only the patient who attempted suicide was withdrawn from the study. All serious adverse events were resolved during the study period (either through surgery or medication), and two cases of serious adverse events (joint swelling and pain) that occurred in the same patient were considered possibly treatment related (Table 1).

Clinically important changes were not observed in electrocardiogram findings, pulse rates, or body temperatures. From baseline to week 52, significant decreases occurred in systolic blood pressure in the TG-C $(p=0.02)$ and placebo groups $(p=$ $0.04)$, and a decrease in diastolic blood pressure occurred in the TG-C group $(p=0.004)$. However, the change in systolic and diastolic blood pressure was not clinically significant. No statistically significant differences were noted in the laboratory test measurements at week 52 compared to baseline, except for one patient who reported clinically 

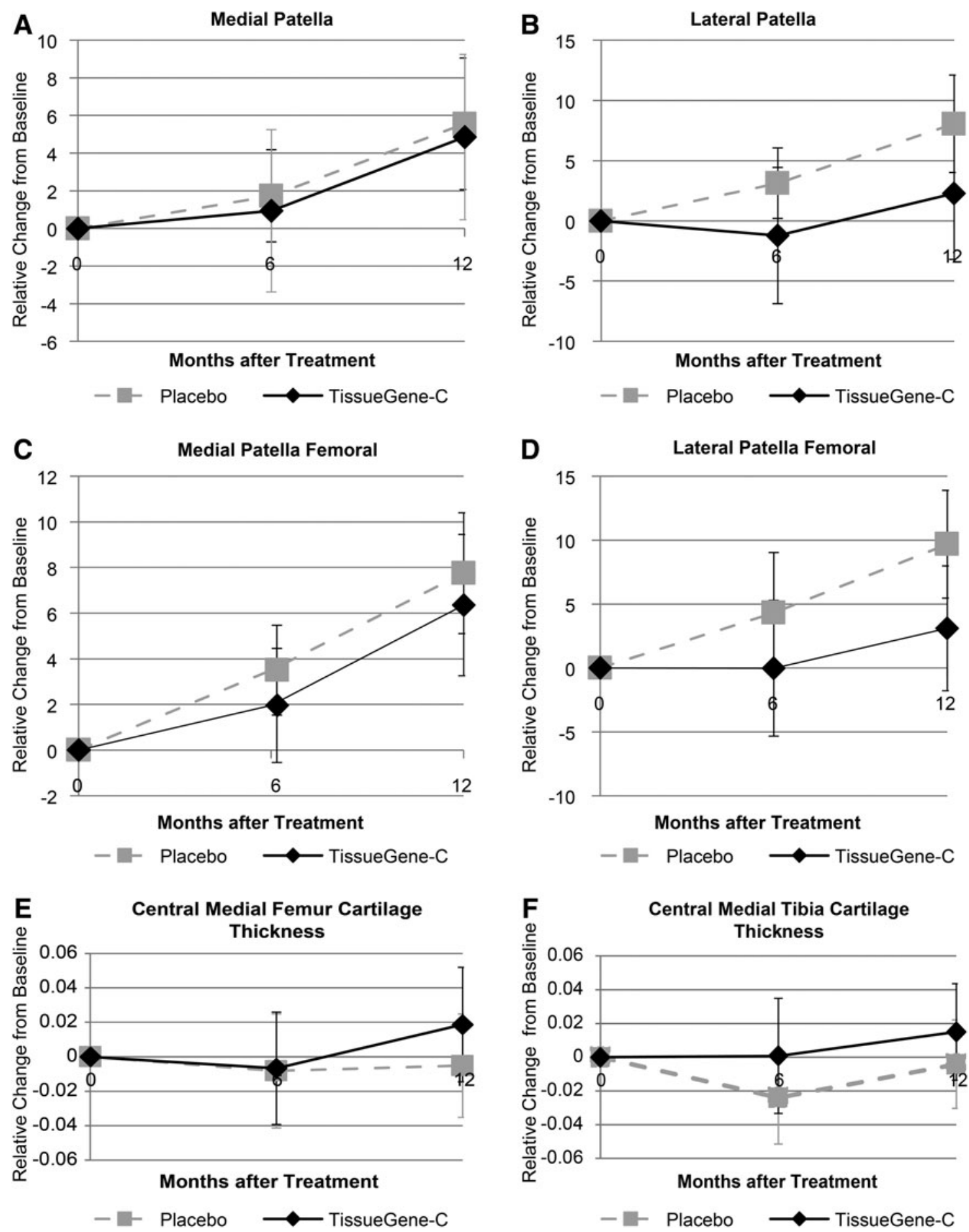

Figure 4. Secondary bone area changes and changes in cartilage thickness. Lateral patella and lateral patella femoral groups showed a clear trend for reduced changes from 26 (6 months) to 52 weeks (12 months) in the TissueGene-C group. At 52 weeks (12 months), changes in both tibia and femur mean cartilage thicknesses in the TissueGene-C treatment group were positive, but these differences were not significantly different.

significant abnormal hyperglycemia at week 52 compared to baseline. However, the investigator considered this event to be unrelated to treatment, as the patient had a high fasting blood sugar value at screening.

\section{DISCUSSION}

Injection of TG-C into the knee joints of osteoarthritis patients resulted in clinically significant improvements in the IKDC and VAS when compared to patients receiving a placebo in terms of the 


\section{A}
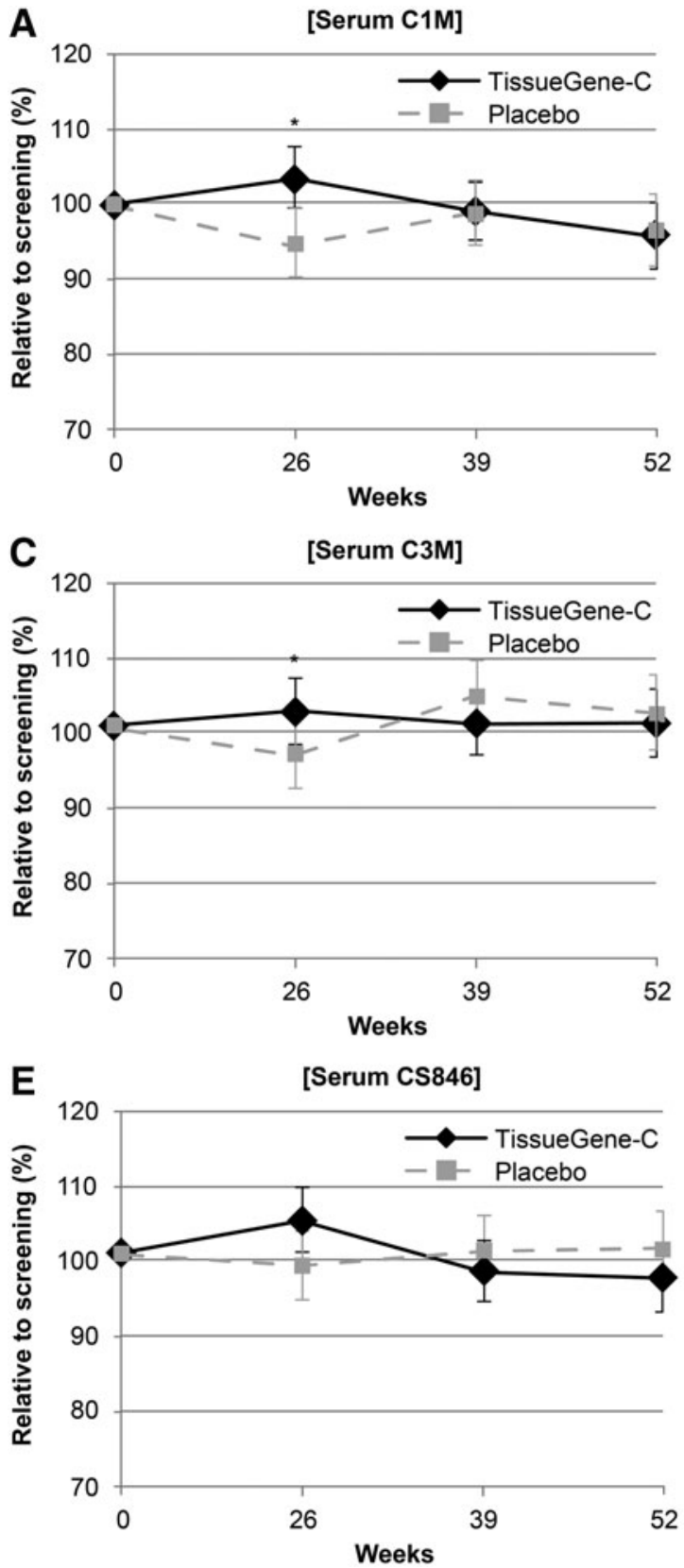
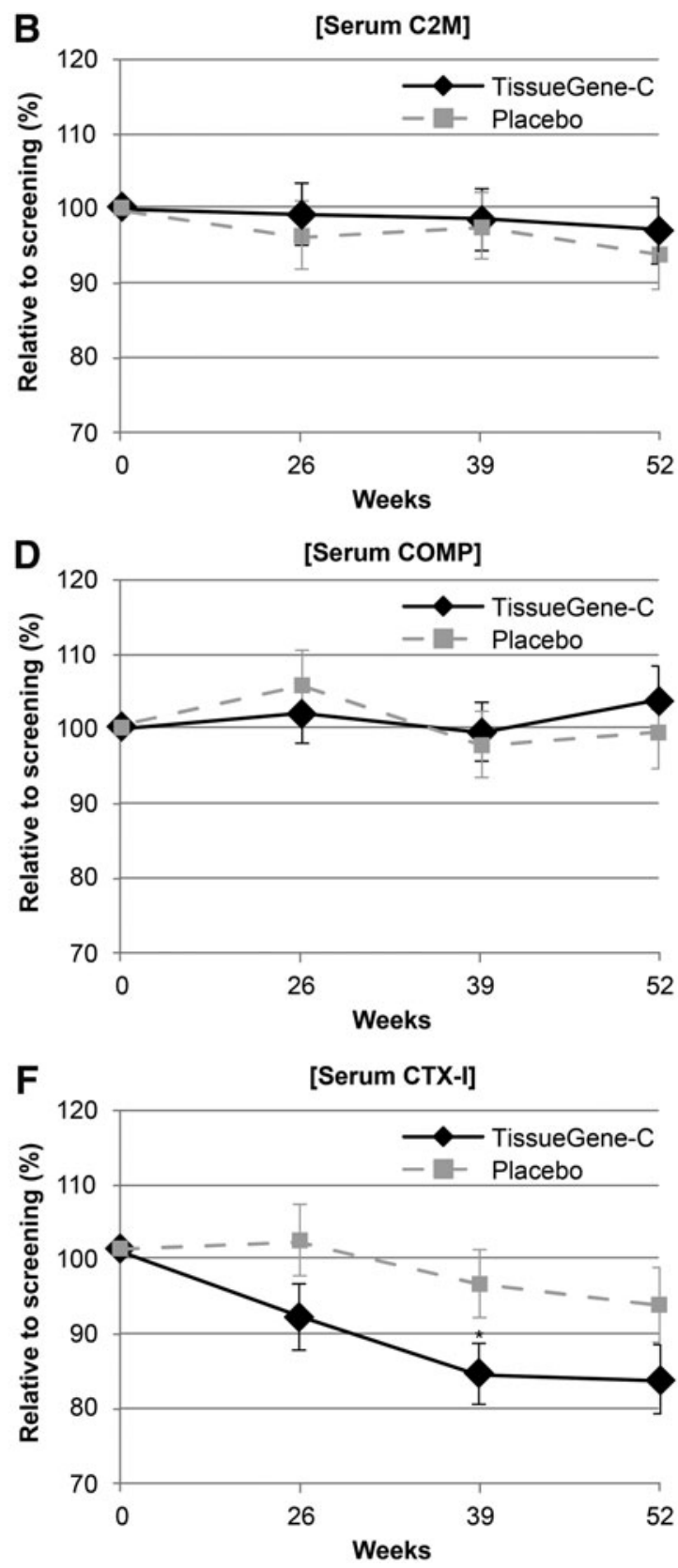

Figure 5. Serum and urine biomarkers. Serum CTX-I levels were statistically significantly lower $(p=0.05)$ with TissueGene-C treatment than with the placebo at 39 weeks. Serum C1M ( $p=0.03)$ and C3M ( $p=0.02)$ levels were transiently significantly higher in the TissueGene-C group than in the placebo group at 26 weeks but not at later time points. The urine CTX-II/creatinine ratio was lower in the TissueGene-C group than in the placebo group at all time points. The data shown are the least-squares geometric mean \pm 1 standard error.

overall subjective assessments of functions, symptoms, and pain after 26,39 , and 52 weeks. Clinically significant improvements were also observed in the WOMAC and KOOS scores. A set of responder criteria for treatment with osteoarthritis drugs has been proposed based on meta-analysis of data from several clinical studies, wherein pain reduction of at least $20 \%$ is clinically meaningful in the context of additional functional improvements. ${ }^{32}$ For symptoms and functions, the primary and most of the secondary efficacy endpoints in this trial were met, with a reduction in pain of $25 \%$ with TG-C treatment after 52 weeks compared to $10 \%$ with the placebo group. These results represent clinically meaningful improvements. Besides, the amount, frequency, and use of rescue drugs did not show any statistically significant difference between the two groups.

The safety of osteoarthritis drugs is of especially high priority because of the high prevalence and 

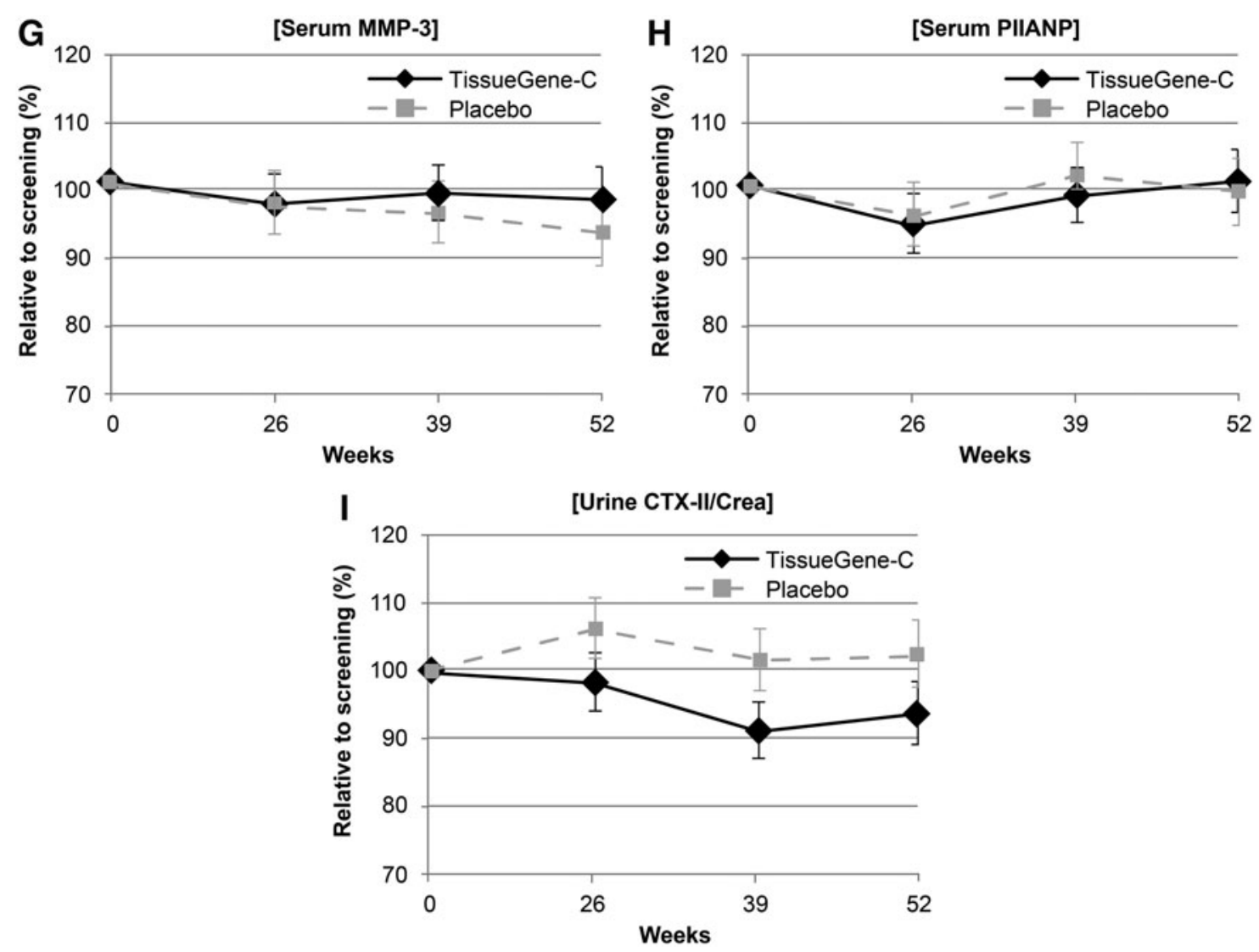

Figure 5. (Continued)

chronic nature of the disease and the frequent presence of comorbidities. The incidences of adverse- and treatment-related events were significantly higher in the TG-C group than in the placebo group. These incidences were mostly related to pain and edema at the injection site, and were largely expected from the TG-C treatment itself. The one case of joint swelling and pain that occurred in the same patient was considered to be possibly treatment related, and these conditions all resolved within the study period.

While the primary goals of this study were to assess patient responses to TG-C, in terms of function and pain, the study was also designed to provide an initial assessment of disease modification by measuring changes in joint space widths (with radiographic evaluation), bone area and cartilage thicknesses (with MRI assessment), and soluble biomarkers. There was a clear trend toward slower bone area progression in the TG-C group, although the difference was not statistically significant due to the limited image quality and statistical power. Interestingly, there was an indication of slower bone area progression in the medial and lateral tibial regions in the TG-C group between 26 and 52 weeks, suggesting the possibility of increasing efficacy over time. Although cartilage thicknesses in the femoral and tibial regions were consistently greater at 52 weeks with TG-C treatment than with the placebo, no statistically significant difference was observed. The lack of statistical significance may stem in part from the observation that many patients had no measurable baseline cartilage.

The observations of lower levels of serum CTX-I and urine CTX-II/creatinine ratios indicated less subchondral bone and cartilage degradation, respectively, in patients treated with TG-C. The transient increase in C1M and C3M levels at the earlier time point may have been due to cartilage regeneration, leading to the release of the 32-mer aggrecan, which binds to Toll-like receptors and can then induce cartilage turnover/inflammation. The 32-mer is the fragment of aggrecan that is generated by MMPs and ADAMTS (a disintegrin and metalloprotease with thrombospondin motifs) proteins. ${ }^{33}$ However, the sample size in this study was not sufficiently large to be conclusive. Hence, 
Table 1. Frequency of adverse and severe adverse events

\begin{tabular}{|c|c|c|c|c|c|}
\hline \multirow[b]{2}{*}{ Variable } & \multicolumn{2}{|c|}{ TissueGene- $\mathrm{C}, \mathrm{N}=78$} & \multicolumn{2}{|c|}{ Placebo, $\mathrm{N}=81$} & \multirow{2}{*}{$\mathrm{p}$-Value } \\
\hline & $\mathrm{n}(\%)$ & Cases & $\mathrm{n}(\%)$ & Cases & \\
\hline Any adverse event & $49(63)$ & 102 & $36(44)$ & 78 & $0.0202^{\mathrm{a}}$ \\
\hline Treatment-related adverse events & $27(35)$ & 39 & $10(12)$ & 14 & $0.0009^{\mathrm{a}}$ \\
\hline Serious adverse events & $11(14)$ & 13 & $0(0)$ & 0 & $0.0003^{b}$ \\
\hline Injury, poisoning and procedural complications & 5 & 5 & 0 & 0 & \\
\hline Clavicle fracture & 1 & 1 & 0 & 0 & \\
\hline Facial bones fracture & 1 & 1 & 0 & 0 & \\
\hline Meniscus injury & 1 & 1 & 0 & 0 & \\
\hline Multiple fractures & 1 & 1 & 0 & 0 & \\
\hline Upper limb fracture & 1 & 1 & 0 & 0 & \\
\hline Nervous system disorders & 2 & 2 & 0 & 0 & \\
\hline Carotid artery stenosis & 1 & 1 & 0 & 0 & \\
\hline Cerebral hemorrhage & 1 & 1 & 0 & 0 & \\
\hline Musculoskeletal and connective tissue disorders & 1 & 2 & 0 & 0 & \\
\hline Arthralgia & 1 & 1 & 0 & 0 & \\
\hline Joint swelling & 1 & 1 & 0 & 0 & \\
\hline Gastrointestinal disorders & 1 & 1 & 0 & 0 & \\
\hline Salivary gland mass & 1 & 1 & 0 & 0 & \\
\hline Neoplasms benign, malignant and unspecified (including cysts and polyps) & 1 & 1 & 0 & 0 & \\
\hline Bladder cancer & 1 & 1 & 0 & 0 & \\
\hline Psychiatric disorders & 1 & 1 & 0 & 0 & \\
\hline Suicide attempt & 1 & 1 & 0 & 0 & \\
\hline Skin and subcutaneous tissue disorders & 1 & 1 & 0 & 0 & \\
\hline Pruritus & 1 & 1 & 0 & 0 & \\
\hline \multicolumn{6}{|l|}{ Serious adverse drug reactions } \\
\hline Musculoskeletal and connective tissue disorders & 1 & 2 & 0 & 0 & \\
\hline Arthralgia & 1 & 1 & 0 & 0 & \\
\hline Joint swelling & 1 & 1 & 0 & 0 & \\
\hline Serious treatment-related adverse events & $1(1)$ & 2 & $0(0)$ & 0 & $0.4906^{b}$ \\
\hline \multicolumn{6}{|l|}{ Adverse events occurring in $\geq 2 \%$ of TissueGene-C-treated patients } \\
\hline Arthralgia & $6(8)$ & 7 & $7(9)$ & 7 & \\
\hline Joint swelling & $5(6)$ & 5 & $5(6)$ & 5 & \\
\hline Back pain & $3(4)$ & 4 & $2(3)$ & 3 & \\
\hline Edema peripheral & 7 (9) & 7 & $1(1)$ & 1 & \\
\hline Injection-site joint pain & $4(5)$ & 4 & $0(0)$ & 0 & \\
\hline Injection-site joint swelling & $3(4)$ & 3 & $0(0)$ & 0 & \\
\hline Injection-site edema & $2(3)$ & 2 & $0(0)$ & 0 & \\
\hline Nasopharyngitis & $3(4)$ & 3 & $3(4)$ & 3 & \\
\hline Headache & $2(3)$ & 2 & $4(5)$ & 11 & \\
\hline Pruritus & $2(3)$ & 4 & $2(3)$ & 2 & \\
\hline
\end{tabular}

Dictionary: MedDRA v18.1.

A serious adverse event was one that resulted in death, threatened the life of a patient, resulted in disability/incapability or a congenital anomaly or birth defect, required hospitalization or extension of a hospital stay, or a major medical event requiring treatment. No serious adverse events were reported in the placebo group.

${ }^{\text {aPearson's }}$ chi-square test.

${ }^{\mathrm{b}}$ Fisher's exact test.

another Phase III study has been planned for the United States with sufficient statistical power to evaluate the DMOAD activity of TG-C.

TG-C was associated with non-statistical but potential structural improvements as much as could be expected from surgically implanted chondrocyte treatment (e.g., Chondron), ${ }^{34}$ which is not expected from the treatment with hyaluronic acid. ${ }^{35}$ Therefore, TG-C is a novel treatment offering sustained improvement of pain and functions for $>1$ year with a single injection and holds for great potential for DMOAD.

\section{ACKNOWLEDGMENTS}

This study was funded by Kolon Life Science (Republic of Korea). The funding source played no roles in the study design, collection, analysis, and interpretation of data; in the writing of the manuscript; or in the decision to submit the manuscript for publication. Trial registration numbers: clinicaltrials.gov NCT02072070 and CRiS: KCT0001112.

\section{AUTHOR DISCLOSURE}

No competing financial interests exist. 


\section{REFERENCES}

1. Vos $T$, Flaxman $A D$, Naghavi $M$, et al. Years lived with disability (YLDs) for 1160 sequelae of 289 diseases and injuries 1990-2010: a systematic analysis for the Global Burden of Disease Study 2010. Lancet 2013;380:2163-2196.

2. Lee $A S$, Ellman MB, Yan D, et al. A current review of molecular mechanisms regarding osteoarthritis and pain. Gene 2013;527:440-447.

3. Cross M, Smith E, Hoy D, et al. The global burden of hip and knee osteoarthritis: estimates from the global burden of disease 2010 study. Ann Rheum Dis 2014;73:1323-1330.

4. McGettigan P, Henry D. Use of non-steroidal antiinflammatory drugs that elevate cardiovascular risk: an examination of sales and essential medicines lists in low-, middle-, and high-income countries. PLoS Med 2013;10:e1001388.

5. Sandell LJ, Aigner T. Articular cartilage and changes in arthritis: an introduction: cell biology of osteoarthritis. Arthritis Res 2001;3:107-113.

6. Van Beuningen HM, Van der Kraan PM, Arntz OJ, et al. Transforming growth factor-beta 1 stimulates articular chondrocyte proteoglycan synthesis and induces osteophyte formation in the murine knee joint. Lab Invest 1994;71:279-290.

7. Yang $X$, Chen $L, X u X$, et al. TGF- $\beta /$ Smad3 signals repress chondrocyte hypertrophic differentiation and are required for maintaining articular cartilage. J Cell Bio 2001;153:35-46.

8. Davidson EB, Van Der Kraan PM, van Den Berg WB. TGF- $\beta$ and osteoarthritis. Osteoarthr Cartil 2007;15:597-604.

9. Moore EE, Bendele AM, Thompson DL, et al. Fibroblast growth factor-18 stimulates chondrogenesis and cartilage repair in a rat model of injury-induced osteoarthritis. Osteoarth Cartil 2005;13:623-631.

10. Lohmander LS, Hellot $S$, Dreher D, et al. Intraarticular sprifermin (recombinant human fibroblast growth factor 18) in knee osteoarthritis: a randomized, double-blind, placebo-controlled trial. Arthritis Rheumatol 2014;66:1820-1831.

11. Dahlberg LE, Aydemir A, Muurahainen N, et al. A first-in-human, double-blind, randomised, placebocontrolled, dose ascending study of intra-articular rhFGF18 (sprifermin) in patients with advanced knee osteoarthritis. Clin Exp Rheumatol 2016;34: 445-450.

12. Freitag J, Bates D, Boyd R, et al. Mesenchymal stem cell therapy in the treatment of osteoarthritis: reparative pathways, safety and efficacya review. BMC Musculoskelet Disord 2016; 17:230.
13. Qi Y, Feng G, Yan W. Mesenchymal stem cellbased treatment for cartilage defects in osteoarthritis. Mol Bio Rep 2012;39:5683-5689.

14. Peterson L, Minas T, Brittberg M, et al. Two- to 9year outcome after autologous chondrocyte transplantation of the knee. Clin Orthop Relat Res 2000;374:212-234.

15. Song SU, Cha YD, Han JU, et al. Hyaline cartilage regeneration using mixed human chondrocytes and transforming growth factor- $\beta 1$-producing chondrocytes. Tissue Eng 2005;11:1516-1526.

16. Ha CW, Noh MJ, Choi KB, et al. Initial Phase I safety of retrovirally transduced human chondrocytes expressing transforming growth factor-beta-1 in degenerative arthritis patients. Cytotherapy 2012;14:247-256.

17. Ha CW, Cho JJ, Elmallah RK, et al. A multicenter, single-blind, Phase lla clinical trial to evaluate the efficacy and safety of a cell-mediated gene therapy in degenerative knee arthritis patients. Hum Gene Ther Clin Dev 2015;26:125-130.

18. Lee MC, Ha CW, Elmallah RK, et al. A placebocontrolled randomized trial to assess the effect of TGF- $\beta 1$-expressing chondrocytes in patients with arthritis of the knee. Bone Joint J 2015;97:924-932.

19. Hefti F, Müller W, Jakob RP, et al. Evaluation of knee ligament injuries with the IKDC form. Knee Surg Sports Traumatol Arthrosc 1993;1:226-234.

20. Hawker GA, Mian S, Kendzerska T, et al. Measures of adult pain: Visual Analog Scale for Pain (VAS Pain), Numeric Rating Scale for Pain (NRS Pain), McGill Pain Questionnaire (MPO), ShortForm McGill Pain Questionnaire (SF-MPO), Chronic Pain Grade Scale (CPGS), Short Form-36 Bodily Pain Scale (SF-36 BPS), and Measure of Intermittent and Constant Osteoarthritis Pain (ICOAP). Arthritis Care Res 2011;63:S240-S252.

21. Kellgren JH, Lawrence JS. Radiological assessment of osteo-arthrosis. Ann Rheum Dis 1957;16: 494-502.

22. van den Borne MP, Raijmakers NJ, Vanlauwe J, et al. International Cartilage Repair Society (ICRS) and Oswestry macroscopic cartilage evaluation scores validated for use in Autologous Chondrocyte Implantation (ACI) and microfracture. Osteoarthr Cartil 2007;15:1397-1402.

23. Bellamy N, Buchanan WW, Goldsmith $\mathrm{CH}$, et al. Validation study of WOMAC: a health status instrument for measuring clinically important patient relevant outcomes to antirheumatic drug therapy in patients with osteoarthritis of the hip or knee. J Rheumatol 1988;15:1833-1840.

24. Roos EM, Roos HP, Ekdahl C, et al. Knee injury and Osteoarthritis Outcome Score (KOOS) valida- tion of a Swedish version. Scand J Med Sci Sports 1998;8:439-448.

25. Charles HC, Kraus VB, Ainslie M, et al. Optimization of the fixed-flexion knee radiograph. Osteoarthritis Cartilage, 2007;15:1221-1224.

26. Bowes MA, Vincent GR, Wolstenholme CB, et al. A novel method for bone area measurement provides new insights into osteoarthritis and its progression. Ann Rheum Dis 2015;74:519-525.

27. Vincent G, Wolstenholme C, Scott I, et al. Fully automatic segmentation of the knee joint using active appearance models. Proceedings MICCAI Medical Image Analysis for the Clinic: A Grand Challenge. North Charleston, SC: CreateSpace, 2010:224-230.

28. Williams TG, Holmes AP, Bowes MA, et al. Measurement and visualization of focal cartilage thickness change by MRI in a study of knee osteoarthritis using a novel image analysis tool. $\mathrm{Br} \mathrm{J}$ Radiol 2010;83:940-948.

29. Hunter DJ, Bowes MA, Eaton CB, et al. Can cartilage loss be detected in knee osteoarthritis (OA) patients with 3-6 months' observation using advanced image analysis of $3 \mathrm{~T}$ MRI? Osteoarthr Cartil 2010;18:677-683.

30. Touraine S, Bouhadoun $H$, Engelke $K$, et al. Influence of meniscus on cartilage and subchondral bone features of knees from older individuals: a cadaver study. PLoS One 2017;12:e0181956.

31. Hunter D, Nevitt M, Lynch J, et al. Longitudinal validation of periarticular bone area and $3 \mathrm{D}$ shape as biomarkers for knee osteoarthritis progression? Data from the FNIH osteoarthritis Biomarkers Consortium. Ann Rheum Dis 2016;75:1607-1614.

32. Pham T, van der Heijde DM, Altman RD, et al OMERACT-OARSI initiative: Osteoarthritis Research Society International set of responder criteria for osteoarthritis clinical trials revisited. Osteoarthritis Cartilage 2004;12:389-399.

33. Lees S, Golub SB, Last K, et al. Bioactivity in an aggrecan 32-mer fragment is mediated via Toll-like receptor 2. Arthritis Rheumatol 2015;67:1240-1249.

34. Kim MK, Choi SW, Kim SR, et al. Autologous chondrocyte implantation in the knee using fibrin. Knee Surg Sports Traumatol Arthrosc 2010;18:528-534.

35. Migliore A, Procopio S. Effectiveness and utility of hyaluronic acid in osteoarthritis. Clin Cases Miner Bone Metab 2015;12:31-33.

Received for publication December 17, 2017; accepted after revision February 26, 2018.

Published online: February 27, 2018. 